\title{
Single leg hop for distance symmetry masks lower limb biomechanics: time to discuss hop distance as decision criterion for return to sport after $\mathrm{ACL}$ reconstruction?
}

\author{
Argyro Kotsifaki (1) , ${ }^{1,2}$ Rod Whiteley 지, 1,3 Sam Van Rossom (ㄷ) ? \\ Vasileios Korakakis 이, ${ }^{1}$ Roald Bahr @ (1) , ${ }^{4,5}$ Vasileios Sideris, ${ }^{1}$ Philip Graham-Smith, ${ }^{6}$ \\ Ilse Jonkers (1) ${ }^{2}$
}

- Additional material is published online only. To view, please visit the journal online (http://dx.doi.org/10.1136/ bjsports-2020-103677).

${ }^{1}$ Rehabilitation Department, Aspetar Orthopaedic and Sports Medicine Hospital, Doha, Qatar ${ }^{2}$ Department of Movement Sciences, Human Movement Biomechanics Research Group, KU Leuven, Leuven, Belgium

${ }^{3}$ School of Human Movement \& Nutrition Sciences, The University of Queensland, Brisbane, Queensland, Australia ${ }^{4}$ Aspetar Sports Injury and Illness Prevention Programme (ASPREV), Aspetar Orthopaedic and Sports Medicine Hospital, Doha, Qatar

${ }^{5}$ Department of Sports Medicine, Norwegian School of Sports

Sciences, Oslo, Norway

${ }^{6}$ Sports Science Department,

Aspire Academy, Doha, Qatar

Correspondence to

Argyro Kotsifaki, Aspeta

Orthopaedic and Sports

Medicine Hospital, Doha 29222,

Qatar;

argyro.kotsifaki@aspetar.com

Accepted 13 February 2021

Check for updates

(c) Author(s) (or their employer(s)) 2021. No commercial re-use. See rights and permissions. Published by BMJ.

To cite: Kotsifaki $A$,

Whiteley R, Van

Rossom $\mathrm{S}$, et al.

Br J Sports Med Epub ahead

of print: [please include Day

Month Year]. doi:10.1136/

bjsports-2020-103677

\section{ABSTRACT}

Background We evaluated the lower limb status of athletes after anterior cruciate ligament reconstruction (ACLR) during the propulsion and landing phases of a single leg hop for distance (SLHD) task after they had been cleared to return to sport. We wanted to evaluate the biomechanical components of the involved (operated) and uninvolved legs of athletes with ACLR and compare these legs with those of uninjured athletes (controls).

Methods We captured standard video-based threedimensional motion and electromyography (EMG) in 26 athletes after ACLR and 23 healthy controls during SLHD and calculated lower limb and trunk kinematics. We calculated lower limb joint moments and work using inverse dynamics and computed lower limb muscle forces using an EMG-constrained musculoskeletal modelling approach. Between-limb (within ACLR athletes) and between-group differences (between ACLR athletes and controls) were evaluated using paired and independent sample t-tests, respectively.

Results Significant differences in kinematics (effect sizes ranging from 0.42 to 1.56$)$, moments ( 0.39 to 1.08), and joint work contribution (0.55 to 1.04) were seen between the involved and uninvolved legs, as well as between groups. Athletes after ACLR achieved a $97 \% \pm 4 \%$ limb symmetry index in hop distance but the symmetry in work done by the knee during propulsion was only $69 \%$. During landing, the involved knee absorbed less work than the uninvolved, while the uninvolved knee absorbed more work than the control group. Athletes after ACLR compensated for lower knee work with greater hip work contribution and by landing with more hip flexion, anterior pelvis tilt, and trunk flexion.

Conclusion Symmetry in performance on a SLHD test does not ensure symmetry in lower limb biomechanics. The distance hopped is a poor measure of knee function, and largely reflects hip and ankle function. Male athletes after ACLR selectively unload the involved limb but outperform controls on the uninvolved knee.

\section{INTRODUCTION}

Functional hop testing is traditionally used to determine readiness to return to sport (RTS) after anterior cruciate ligament reconstruction (ACLR). ${ }^{12}$ Typically, functional symmetry between legs is assessed with a hop test battery. ${ }^{34}$ The primary hop tests are in forward direction and the outcome of interest is distance in three of them-single hop, triple hop and cross-over hop for distance. ${ }^{5}$ With these tests, Limb Symmetry Index (LSI) of $>90 \%$ in distance hopped is recommended as a cut-off point for safe RTS. $^{6}$ The use of $>90 \%$ LSI thresholds for functional hop tests has previously been questioned because of the potential risk of masking information about movement deficits and different strategies employed. $^{78}$

Despite the advances in ACLR techniques and the use of criteria to RTS, graft failure and contralateral ACL injury risk on returning to sport are reported as $9 \%-19 \%$ and $18 \%-22 \%$, respectively. ${ }^{9-11}$ In addition, more than a third of individuals after ACLR are unable to return to pre-injury levels of activity, ${ }^{12}$ justifying the high burden of an ACL injury. ${ }^{13}$ More recent evidence indicates that re-injury rates can be reduced by $50 \%$ for every month RTS is delayed up to 9 months, with no further reductions in risk shown after this point. ${ }^{3}$ After ligamentisation, it can be assumed that the reconstructed ACL has sufficient integrity to cope with sporting demands. The strength of the ACL in the uninjured leg should similarly be capable of withstanding normal sporting loads. These high failure rates therefore suggest that these patients experience abnormally high, potentially injurious loads in the previously uninjured leg which eventually causes injury.

The single leg hop for distance (SLHD) is most frequently reported ${ }^{14}$ and has been argued to be the only 'clinician-friendly' test able to evaluate knee function after ACLR. ${ }^{15}$ During a SLHD test, despite achieving symmetry in hop distance performance, athletes after ACLR still present between-limb kinematic and kinetic asymmetries ${ }^{16}$ which might explain the unacceptably high rate of secondary injuries after RTS. Distance hopped during a performance test, such as the SLHD, indirectly assesses the total work done by the athlete's lower leg kinetic chain, specifically the hip, knee and ankle joints during the propulsion phase. ${ }^{17}$ The work done during the propulsion phase of the hop then needs to be absorbed by the same limb during the landing phase. Examining the work during propulsion and landing, as well as the contribution of the different joints may reveal motor strategies associated with 
aberrantly high, potentially injurious, loads. The relative contributions of each part of the kinetic chain likely vary depending on the individual's motor strategy. Protectively off-loading one joint might result in higher compensatory loading at another.

For all the above-stated reasons, an in-depth biomechanical analysis of the movement quality during the SLHD is imperative to conclude on its suitability to identify movement deficits or compensatory strategies after ACLR. Accordingly, we sought to describe in depth the biomechanical performance (kinematics, kinetics, work, and individual muscle forces) of athletes at the time they had met all criteria for RTS and compare with healthy controls during the propulsion and landing phases of the SLHD. The objective of this study was to evaluate biomechanical components during the propulsion and landing phases of the SLHD test in athletes with ACLR who had passed criteria for RTS, compared with contralateral knees and uninjured athletes.

\section{METHODS}

\section{Study design and setting}

This laboratory study involved a case-control comparative analysis of an ACLR and a healthy cohort. All participants provided informed consent.

\section{Participants, inclusion and exclusion criteria}

We included 49 male participants in this study, 26 eligible patients after primary ACLR and 23 control subjects (figure 1, table 1). Patients with ACLR were enrolled after completion of a standardised rehabilitation protocol in our hospital and after receiving clearance to RTS. The RTS process employs a shared decision making strategy ${ }^{18}$ which includes consideration of key RTS criteria including (1) clearance by both their surgeon and physiotherapist, (2) completion of a sports specific on-field rehabilitation programme, (3) quadriceps strength LSI $>90 \%$, and (4) hop test battery LSI $>90 \% 0 .{ }^{4}$ ACLR individuals were athletes (preinjury Tegner score $\geq 7$ ) aged between 18 and 35 years with a complete, unilateral ACL injury, either with an autologous ipsilateral bone-patellar-tendon-bone or a hamstrings graft (semitendinosus and gracilis) as clinically decided by the treating surgeon and athlete. Subjects with concomitant meniscal injury that did not significantly impede the rehabilitation course were also included in the study. Participants were excluded if they had concomitant grade III knee ligament injury (other than ACL), full thickness articular cartilage lesion, history of other lower extremity surgery (in either leg), back pain or lower extremity

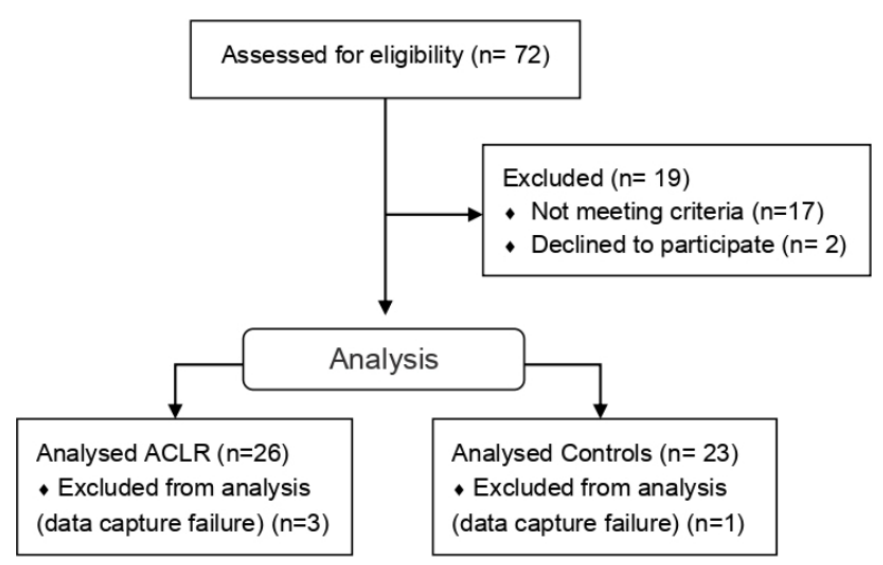

Figure 1 Study flow diagram. ACLR, anterior cruciate ligament reconstruction.

\begin{tabular}{lclc}
\hline Table 1 & Participants information & & \\
\hline & $\begin{array}{l}\text { ACLR group } \\
\text { ( } \mathbf{n}=26)\end{array}$ & $\begin{array}{l}\text { Control group } \\
(\mathbf{n}=23)\end{array}$ & P value \\
\hline Age (years) & $23.2 \pm 3.4$ & $28.3 \pm 4.4$ & $<0.001$ \\
\hline Body mass (kg) & $71.4 \pm 12.1$ & $76.1 \pm 7.4$ & 0.10 \\
\hline Height (cm) & $173(166$ to 182)* & $178.2 \pm 6.9$ & 0.18 \\
\hline Body mass index (kg/m $\left.{ }^{2}\right)$ & $23.3 \pm 2.3$ & $23.9 \pm 1.6$ & 0.24 \\
\hline Tegner score pre-injury & $9(9 \text { to } 9)^{*}$ & $7(7 \text { to } 9)^{*}$ & $<0.001$ \\
\hline IKDC & $94.9 \pm 7.0$ & 100 & 0.002 \\
\hline ACL-RSI & $92.0 \pm 10.6$ & NA & NA \\
\hline Quadriceps strength LSI \% & $94 \pm 6$ & NA & 0.011 \\
\hline SLHD LSI \% & $97 \pm 4$ & $100 \pm 5$ & 0.07 \\
\hline TRHD LSI \% & $97 \pm 5$ & $100(98 \text { to } 102)^{*}$ & NA \\
\hline Return to sport (months) & $9.5 \pm 2.7$ & NA & \\
\hline ACL hamstrings autograft, & $10(38)$ & & \\
$\mathrm{n}$ (\%) & & & \\
\hline Isolated ACL injury, $\mathrm{n}$ & 17 & & \\
\hline Meniscal injury, $\mathrm{n}$ & 12 & & \\
\hline Cartilage lesion, $\mathrm{n}$ & 2 & & \\
\hline All & & & \\
\hline
\end{tabular}

All participants were male. Values other than number of participants are expressed as mean \pm SD except where the data were not normally distributed where these data are presented as median and IQR. Independent-sample $t$ tests were used for between groups comparison, significant difference $(p<0.05)$.

${ }^{*}$ Not normally distributed data.

ACLR, anterior cruciate ligament reconstruction; ACL-RSI, Anterior Cruciate Ligament-Return to Sport; IKDC, International Knee Documentation Subjective Knee questionnaire; LSI, Limb Symmetry Index; NA, not available; SLHD, single leg hop for distance; TRHD, triple hop for distance.

injury (other than primary ACL) in the prior 3 months. Twentythree male control participants were also recruited by contacting healthcare providers and sports club doctors. Inclusion criteria were an age of $18-35$ years, Tegner score $\geq 7$, participation in level I or II sports three times a week or more, and no history of musculoskeletal injury of the lower limb 3 months prior to testing.

\section{Equipment, participant preparation and marker set}

Forty-two reflective markers were placed according to a fullbody Plug-in-Gait marker-set, extended with additional anatomical markers on the sacrum, medial knee and medial ankle. ${ }^{19}$ Three marker clusters replaced the single maker laterally on each thigh and shank. ${ }^{20}$ The markers' motion was captured with a 14-camera motion capture system (Vicon, Oxford, UK, $250 \mathrm{~Hz}$ ). Ground reaction forces (GRFs) were collected synchronously with marker trajectories using five ground-embedded force plates (Kistler, Switzerland, $1000 \mathrm{~Hz}$ ). Electromyographic (EMG) activity was collected simultaneously (Delsys Myomonitor IV, USA, $2000 \mathrm{~Hz}$ ) from the vastus lateralis and medialis, rectus femoris, biceps femoris, semitendinosus, medial and lateral gastrocnemius, and tensor fasciae latae (TFL). ${ }^{21}$

\section{Experimental setup, procedure and testing}

All participants were evaluated at the same location by the same examiner and wore athletic shorts and standard shoes. They performed a 7 min warm up session including running, side running, deep squats and double leg jumps. Then they performed a series of maximal voluntary isometric contractions to obtain maximum EMG values for each measured muscle. The first MVIC was performed to familiarise the participant with the procedure. The MVIC was assessed for TFL in standing, for quadriceps in a sitting position ( $60^{\circ}$ of knee flexion), for hamstrings in prone position (knee flexion at $30^{\circ}$ ) and for gastrocnemius in 
standing. Each subject performed two maximum effort isometric contractions of each muscle group, where each lasted $5 \mathrm{~s}$ with $30 \mathrm{~s}$ rest between each trial. ${ }^{22} 23$ Test limb order was randomised using a coin toss.

Subsequently, participants practised SLHD until they felt comfortable, while verbal feedback on their performance was provided. For the execution of SLHD, participants stood on a force plate, placed their hands over their hips and started from an upright single leg standing position before countermoving to a self-selected depth and then jumped horizontally as far as possible, landing on the same leg. Data were collected bilaterally during four successful trials. A successful trial required participants to hold the final landing for at least $2 \mathrm{~s}$. Test limb order was also randomised. Limb dominance was determined by asking the participants with which limb they would prefer to kick a ball. ${ }^{24}$

\section{Data processing}

Data were processed in Visual 3D (C-Motion, Germantown, MD). Marker trajectories and GRFs were low-pass filtered using a zero-lag, fourth order, Butterworth filter with the same $15 \mathrm{~Hz}$ cut-off frequency. Propulsion phase was defined as $0.4 \mathrm{~s}$ prior to take off until take off-to include hip concentric phase which occurs before peak knee flexion, and landing phase from initial contact to peak knee flexion.

Joint angles were calculated using a Visual 3D hybrid model with a Cardan X-Y-Z (mediolateral, anteroposterior, vertical) rotation sequence. ${ }^{25}$ Ankle, hip, and knee joint angles were defined as the angle between the distal and the proximal segment. Pelvis was defined using the Coda model. ${ }^{26}$ Pelvis and trunk segment angles were determined with respect to the global coordinate system. Joint internal moments were resolved in the proximal coordinate system. We calculated kinematics for trunk and pelvis, and also kinematics and kinetics for hip, knee, and ankle joints, bilaterally. The variables of interest were: peak joint angles, joint angles at toe-off and initial contact, peak internal joint moments. For lower limb joints, work generation and absorption were determined as the time integral of the net positive and negative joint power, respectively. Joint power was calculated by using all three components. The joint contribution was then determined as a percentage contribution to the summed work of all three lower limb joints during the propulsion and landing phases. Work and joint moments were normalised to body mass. LSI was determined as the percentage of the involved limb divided by the uninvolved limb. ${ }^{5}{ }^{14}$ All variables were extracted for the propulsion and landing phase separately.

\section{Muscle force calculation}

We used a generic musculoskeletal model for deep squatting ${ }^{27}$ and followed a standard musculoskeletal modelling workflow implemented in Opensim V.3. $3^{28}$ to calculate muscle forces. We included knee varus, valgus, and knee internal-external rotation degree of freedom, and we defined the major knee ligaments following Xu et al, ${ }^{29}$ using host mesh fitting for registering origin and insertion points between models. The maximum isometric force of each muscle was multiplied by 3 to allow generation of high forces required to perform the dynamic movements. ${ }^{3031}$ The foot was modelled as one rigid segment. An EMG-constrained static optimisation approach that omitted the force-length and force-velocity behavior ${ }^{32}$ was used to determine the muscle forces balancing the external joint moments. For this, EMG signals were first processed with a $20-400 \mathrm{~Hz}$ bandpass fourth order Butterworth filter, then rectified and filtered with a $10 \mathrm{~Hz}$ low-pass second order Butterworth filter, and finally, normalised to the peak processed EMG value measured for the subject across all activities performed during data collection, which included maximum voluntary contractions, running, jumping, cutting, and hopping for maximum distance. ${ }^{33}$ To account for participant-specific mass, muscle forces were normalised to body weight. Finally, we calculated the contribution of the individual muscle force impulse during the SLHD tests as a percentage of the overall muscle force impulse (30 in total for each leg), for landing and propulsion phase separately.

\section{Power analysis}

As there are no data comparing work done for ACLR injured, uninjured and comparison groups we were unable to conduct an a priori power analysis to determine our sample size. Based on previous research examining biomechanics of similar cohorts, ${ }^{3435}$ we arbitrarily planned for a sample size of 25 per group.

\section{Statistical analysis}

Descriptive statistics were used to summarise the characteristics of the participants and measurements, using the average of the four trials collected. Normality of distribution of data was checked using Shapiro-Wilk normality tests. ${ }^{36}$ There were no outliers in the data, as assessed by inspection of a boxplot and there was homogeneity of variances, as assessed by Levene's test for equality of variances. ${ }^{37}$ Preliminary between-limb analyses of the control group for all parameters of interest indicated no significant differences, therefore, dominant and non-dominant limbs were combined and included independently for subsequent comparisons with the injured cohort. In order to ensure this approach was valid, the analysis was rerun using only one (randomly selected) control limb from each control subject with no meaningful change observed in outcomes (online supplemental file 1). We used paired-samples t-tests for between-limb analyses and independent $t$-tests for between-group analyses. Bonferroni adjustment of $\mathrm{p}$ values was performed to adjust for the post hoc comparisons $(p<0.017)$. Non-normally distributed data were compared using non-parametric tests; Wilcoxon for between limbs ${ }^{38}$ and Mann-Whitney for between groups ${ }^{39}$ comparisons. Effect sizes were calculated using the pooled ${ }^{40}$ (between-limb) and the pooled weighted ${ }^{41}$ (between-group) SD. Values of $0.2,0.5$ and 0.8 were used as the lower thresholds for small, moderate, and large effects, respectively. ${ }^{40}$ All analyses were performed using SPSS V.26, (IBM).

\section{RESULTS}

Participants were tested within 2 weeks of clearance to RTS at $9.5 \pm 2.7$ months following surgery. The ACLR group achieved a $97 \% \pm 4 \%$ LSI and the control group $100 \% \pm 5 \%$ LSI in hop distance during the SLHD. Hop distance normalised to leg length (ASIS to lateral malleolus) was $1.76 \pm 0.14,1.82 \pm 0.16$ and $1.79 \pm 0.16$ for the involved limb, uninvolved limb and control group, respectively. Kinematics, kinetics, and joint contribution to total work done results, for athletes after ACLR and the control group are presented in table 2 .

\section{Propulsion phase}

During propulsion, the involved knee generated significantly less work than the uninvolved $(\mathrm{d}=1.08, \mathrm{p}<0.001)$ and the control group $(\mathrm{d}=0.63, \mathrm{p}=0.010)$ (figure 2 ). In terms of joint contribution to the total work, the involved had less contribution from the knee and significantly more from the hip than the uninvolved limb (figure 3). In addition, more hip and knee flexion in the involved than the uninvolved limb and controls was seen at 


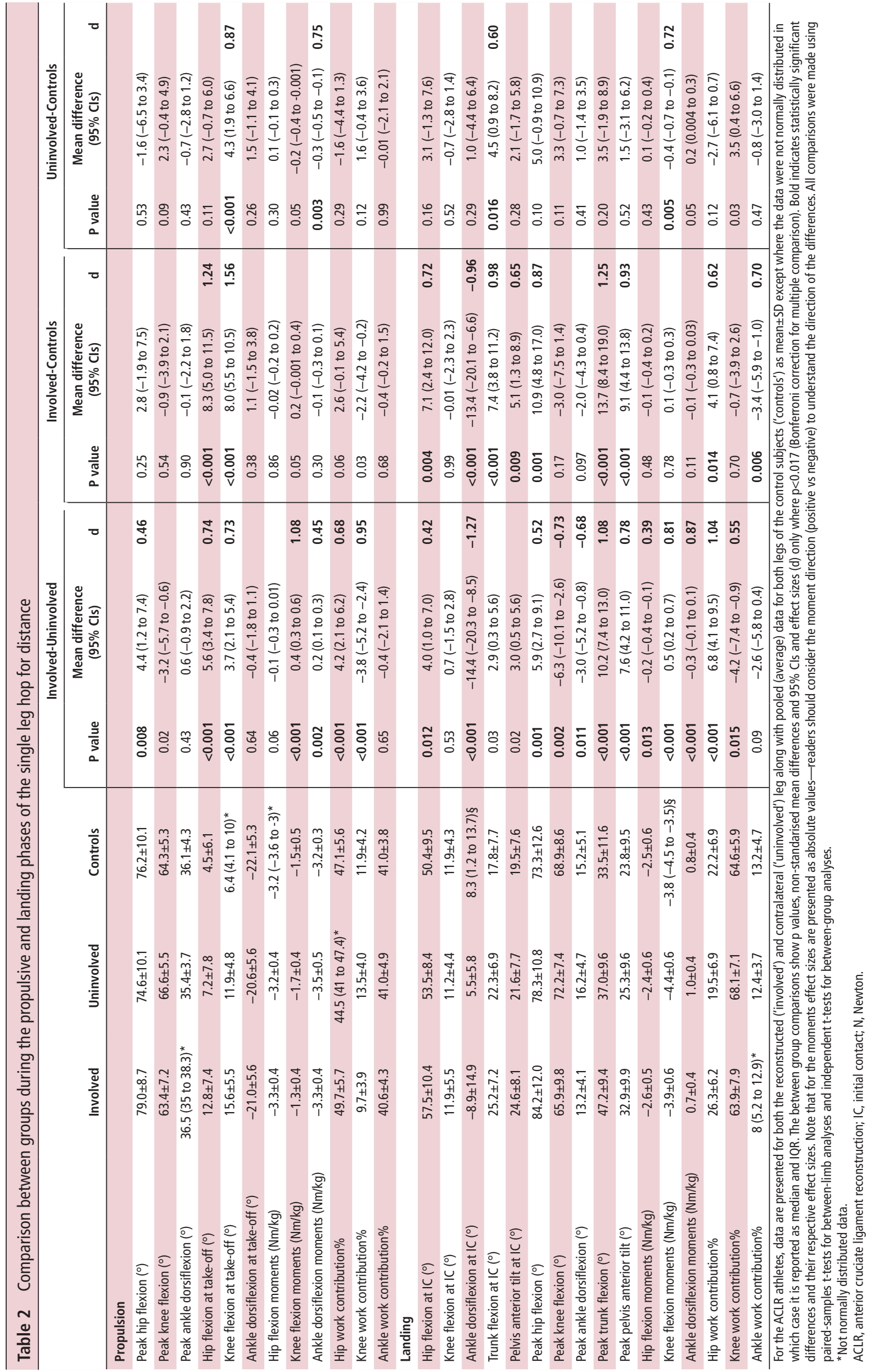




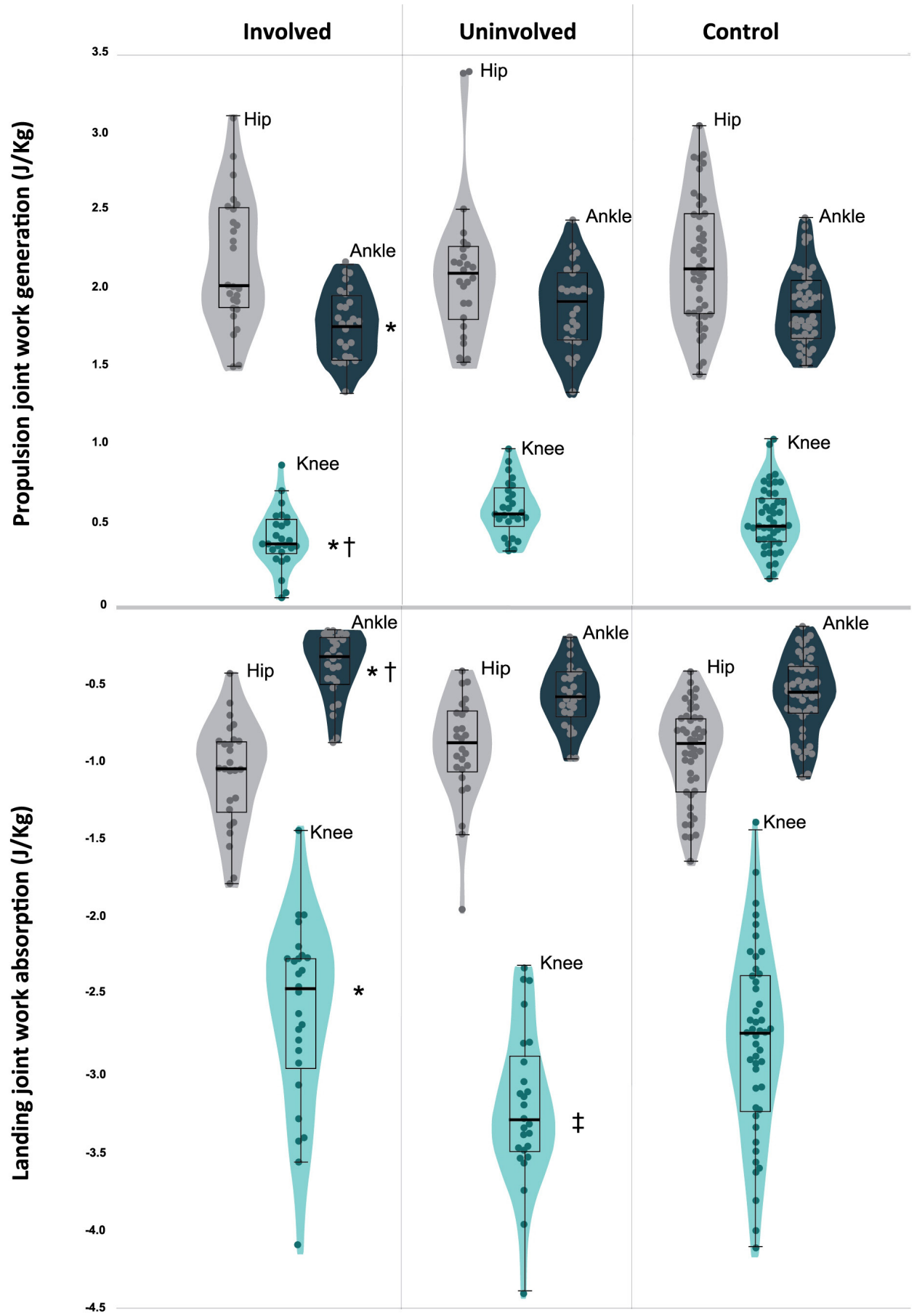

Figure 2 Hip, knee, and ankle work during propulsion and landing of the single hop for distance task for the involved limb, the uninvolved limb and controls. Violin plots represent the smoothed distribution of the data. Box plots represent the distribution of the work values for each joint. Work is positive during propulsion and higher positive values represent more work generation. Work is negative during landing, so higher negative values represent more work absorption. *Significant difference involved-uninvolved, †Significant difference involved-controls, $¥$ Significant difference uninvolved-controls.

take-off. Regarding muscle force distribution, the involved limb had a greater contribution of the lateral hamstrings $(d=0.93$, $\mathrm{p}=0.001)$ and soleus $(\mathrm{d}=0.76, \mathrm{p}=0.003)$ than the controls and lower gluteus medius contribution $(d=0.81, p=0.002)$ than the uninvolved limb (figure 4).

\section{Landing phase}

During landing, the uninvolved knee had more work done than the involved knee $(\mathrm{d}=1.12, \mathrm{p}<0.001)$ and the control group $(d=0.76, p=0.003)$, whereas the involved ankle did less work than both the uninvolved $(\mathrm{d}=0.96, \mathrm{p}=0.007)$ and the controls $(\mathrm{d}=0.77, \mathrm{p}=0.001)$ (figure 2). The involved limb presented with more hip flexion and ankle plantarflexion compared with the uninvolved and controls at initial contact. Furthermore, the involved limb had higher peak hip flexion, peak pelvis anterior tilt and peak trunk flexion than the uninvolved and control subjects and lower peak knee flexion than the uninvolved limb. Regarding muscle forces, the involved limb had greater contribution of lateral hamstrings and medial gastrocnemius than the uninvolved $(\mathrm{d}=1.02, \mathrm{p}<0.001$ and $\mathrm{d}=0.68, \mathrm{p}=0.007$, respectively) and controls $(\mathrm{d}=1.07, \mathrm{p}<0.001$ and $\mathrm{d}=0.94, \mathrm{p}=0.004$, 


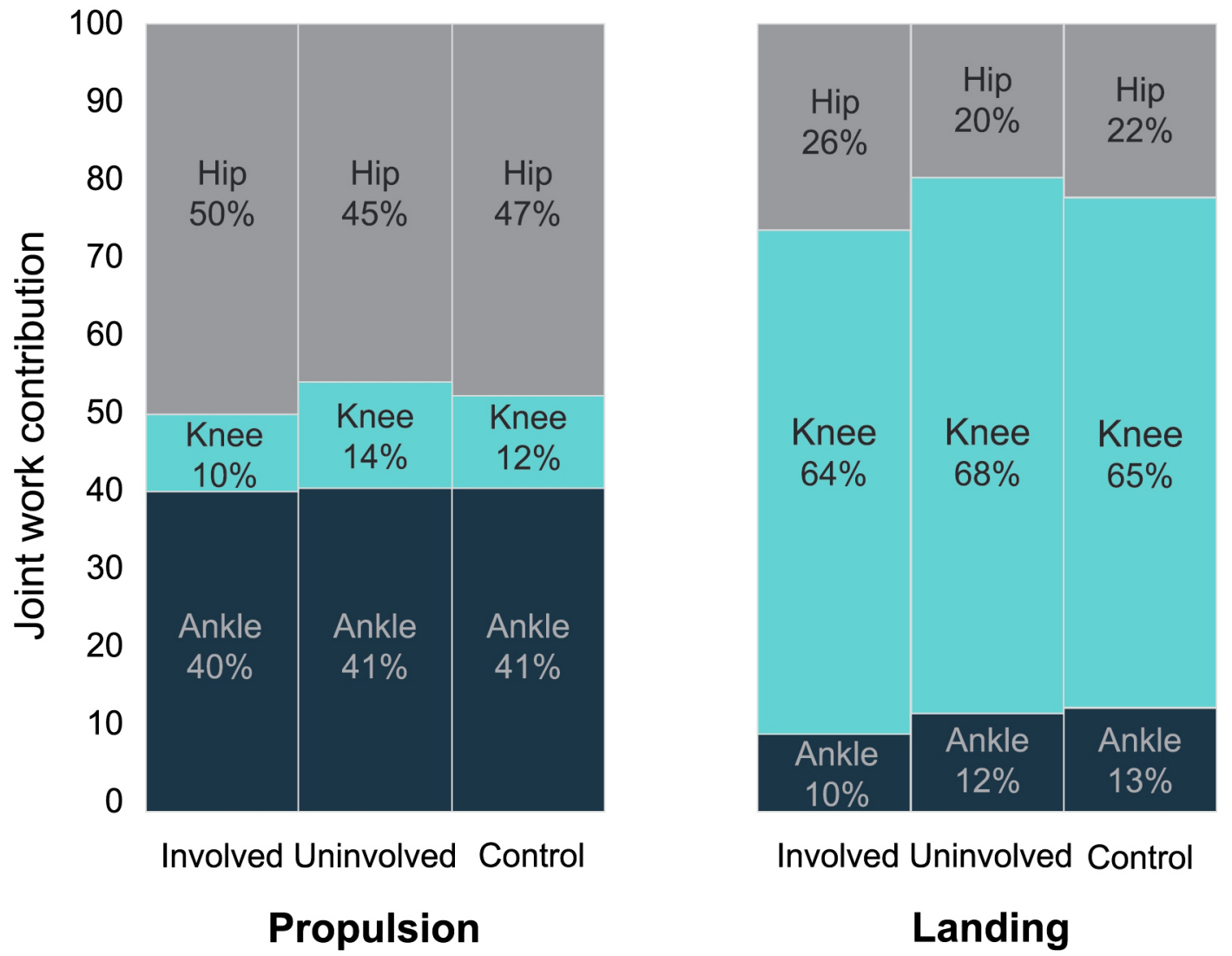

Figure 3 Average percentage work contributions from the hip, knee, and ankle joints during the propulsion and landing phases of the single leg hop for distance. During propulsion, hip and ankle predominantly drive the movement, while knee joint has only a small contribution. During landing the knee joint is the main work absorber performing $65 \%$ of the total work. The involved knee has less contribution in both phases, with compensatory increases at the hip joint.
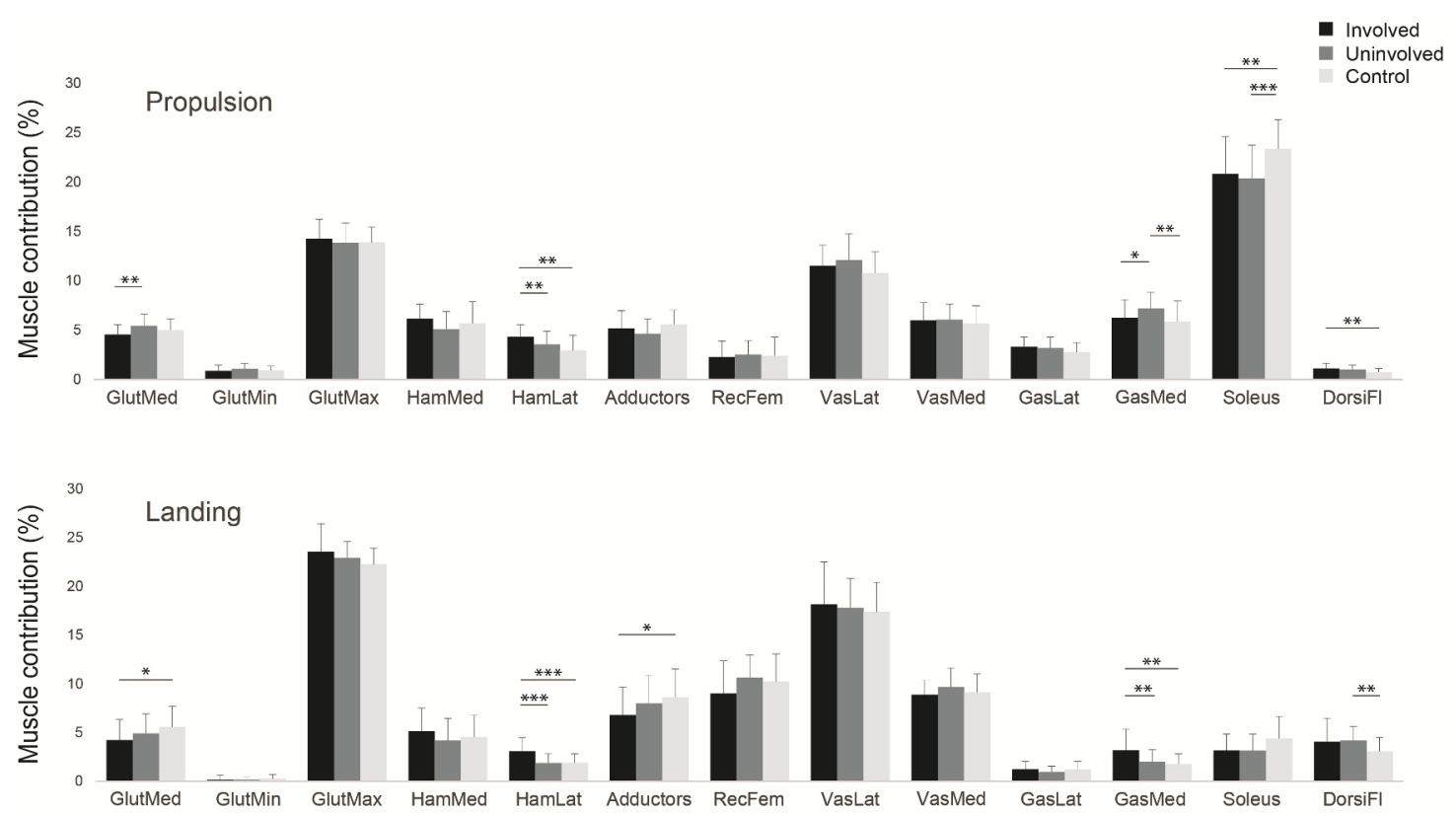

Figure 4 Individual muscle forces impulse contribution for the involved leg (black), the uninvolved (grey) and the controls (white), during the propulsive (top) and landing phases (bottom) of the single leg hop for distance. ${ }^{*} P<0.05,{ }^{*} p<0.01,{ }^{* *} p<0.001$. From left to right: gluteus medius, gluteus minimus, gluteus maximus, medial hamstrings, lateral hamstrings, hip adductors, rectus femoris, vastus lateralis, vastus medialis, gastrocnemius lateral head, gastrocnemius medial head, soleus, dorsiflexors. 
respectively), and less contribution of gluteus medius and adductors than controls (figure 4).

\section{DISCUSSION}

Despite passing strict testing criteria to RTS, differences in biomechanics were identified between the injured and uninjured limbs of athletes after ACLR, as well as with the healthy comparison group.

Hop distance as a metric of knee function needs reappraisal Athletes after ACLR were almost symmetrical in terms of hop distance (97\%), but they still exhibited moderate to large differences in knee function. The ACLR group achieved 69\% symmetry in knee work done during propulsion. This relatively large between-leg difference is more easily understood when we recognise that the knee joint contributes little (about 12\%) to the propulsive phase of a SLHD. With $88 \%$ of the distance hopped being determined by the hip and ankle joint, deficits at the knee joint are easily missed. Simply put: the distance hopped is a poor measure of knee joint function, and largely reflects hip and ankle function (figure 3). Consequently, it may not be the appropriate metric to use for testing an athlete with a knee injury and its relevance is questionable.

Our data supplements previous arguments that questioned SLHD as a metric of knee function in ACL injured athletes. While hop distance has been correlated with self-reported measures of knee function ${ }^{42}$ and fear avoidance, ${ }^{43}$ there are more appropriate tools to measure these patient-reported outcomes. ${ }^{445}$ Clinicians have used hop tests to estimate lower limb muscular strength, ${ }^{46}$ although they are seen to have a low correlation with quadriceps strength: aspects of knee strength can explain 13\%-25\% of the variance of horizontal hop performance. ${ }^{47-49}$ Symmetry in hop distance is achieved earlier during rehabilitation than symmetry in isokinetic knee strength ${ }^{14}{ }^{47}$ suggesting that emphasising hop distance LSI risks overestimating rehabilitation status. ${ }^{50}$ Furthermore, hop tests are still being used as criteria to RTS, not due to specificity, but mainly because passing a battery of tests is associated with lower risk of reinjury. ${ }^{34}$ However, a more detailed analysis of the data indicates that only strength measures were associated with reinjury rates and not the hop tests ${ }^{34}$ and its predictive validity cannot be established based on available literature. ${ }^{51}$ In accordance with our results, the use of $>90 \%$ LSI thresholds for functional hop tests has previously been questioned because of the potential risk of masking information about movement deficits and different strategies employed. ${ }^{78}$ Our results add that the knee's contribution is limited in achieving the outcome parameter (hop distance) which is largely a function of the hip and ankle joints.

\section{Differences in loading}

During the landing phase, knee joint work and work contribution (\%) were not different between the involved knee and the control group; however, knee work was significantly larger in the uninvolved knee compared with the involved leg and control group. This finding is in agreement with previous research showing higher loading of the uninvolved healthy limb compared with a control group for performance and knee strength, ${ }^{52}$ as well as for knee extension moments ${ }^{53}$ in individuals after ACLR at the time of RTS. Similar findings are reported also in other tasks including drop vertical jump, ${ }^{54}$ gait and stair ambulation, ${ }^{55}$ in both male and female populations, ${ }^{52-55}$ indicating that task or gender might not have an influence. A possible explanation might be the comprehensive rehabilitation protocol employed (that addressed loading of not only the involved limb but also the uninvolved limb), improved the deconditioning commonly seen in individuals after ACLR. ${ }^{756}$ However, caution is needed with an adoption of a movement pattern that shifts the mechanical demands away from the reconstructed knee during bilateral tasks, ${ }^{57}$ and towards overuse of the contralateral limb during the long rehabilitation period as a compensatory and protective mechanism. ${ }^{55}$ This maladaptive asymmetry might place the intact contralateral leg at an increased risk for injury compared with the involved leg and may offer a possible explanation for contralateral ACL injuries ${ }^{58}$ and for the higher post-traumatic osteoarthritis prevalence in the contralateral knee. ${ }^{59}$

\section{Whole-body adaptations}

Athletes after ACLR deployed a whole-body adaptation strategy to account for the between limb difference in knee function. During the landing phase, the knee and ankle contributed less to the total work on the involved leg, with more work observed at the hip. The total energy absorption was less in the involved leg than the controls, by adopting a different upper body compensatory mechanism to reduce lower limb loading. Individuals landed on the involved limb with more hip flexion, anterior pelvis tilt, and trunk flexion maintaining a more extended knee position-a mechanism commonly seen in individuals after ACLR to reduce the knee load. ${ }^{5053} \mathrm{At}$ initial contact, individuals preferred to land with a forefoot strike with their involved leg instead of using a heel strike pattern, probably as an additional technique to reduce knee loading. ${ }^{6061}$ There are conflicting findings in the literature for the intralimb adaptations. Some patients used their hips as a compensatory strategy, ${ }^{35}$ while others offloaded the knee compensating at the ankle. ${ }^{3435}$

Differences between limbs were also found for muscle function and percentage contributions. The ACLR group had greater hamstrings contribution on the involved leg, acting as a protective mechanism. Hamstrings have a posterior line of pull in a flexed knee and might thus act as an ACL agonist, counteracting high anterior tibial shear forces. ${ }^{62} 63$

\section{Clinical implications}

Hop distance symmetry is commonly used as a criterion for an athlete to RTS after ACLR. The results of this study indicate that during a SLHD, symmetry in hop distance does not ensure symmetry in lower limb biomechanical parameters, especially for the knee joint. Future research might explore if these results can be generalised to the other common tests like the triple hop ${ }^{64}$ and the cross-over hop for distance and whether they measure similar constructs. Measuring hop distance, or its symmetry, risks overestimating rehabilitation status in terms of the knee joint, may lead to erroneous progression, and offers little additional clinical information in the RTS-readiness decision. Conversely, the SLHD task, and specifically the landing phase, can offer valuable information on the status of the knee joint, but requires three-dimensional biomechanical analysis-an apparatus not frequently available in clinical setting. Consequently, future research should focus on identifying proxy variables of these loading measures, perhaps from wearable technology or other approaches, which would allow monitoring these parameters in a clinical setting. In the absence of this technology, we suggest tasks other than SLHD, and variables other than hop distance may be more appropriate to capture the differences between legs and offer a more accurate estimation of the status of the knee. 


\section{What are the findings?}

- Hop distance may not be the appropriate metric to use for testing male athletes with a knee injury.

- Male athletes after anterior cruciate ligament (ACL) reconstruction during landing underload their reconstructed knee by compensating mostly at their hip.

- The tendency to overload the uninjured knee compared with healthy controls might explain the increased rates of contralateral injuries.

\section{How might it impact on clinical practice in the future?}

- Our findings suggest that symmetry in hop distance may not mean knee function is also symmetrical. Thus, using hop distance as a discharge criterion in male athletes after $\mathrm{ACL}$ reconstruction may not be appropriate.

\section{Methodological considerations}

The recruitment of only males from a single site suggests interpretation of these results with caution in females and other populations. Importantly, we note that there were baseline differences in age (5 years older) and activity level (1.3 lower on the Tegner scale) for our control subjects compared with the professional athletes in the ACLR cohort. We did not adjust for these differences during the analyses and these findings should be extrapolated and generalised with caution until such confirmatory research has been done. We did not measure quadriceps strength in the control group. However, it is assumed that they were symmetrical (>90\% LSI) for the quadriceps strength. ${ }^{65}$ Although musculoskeletal modelling allows for the prediction of in vivo muscle forces without invasive methods, it is not without limitations. Since measuring muscle forces in vivo during jumping is not feasible, we do not have a direct test for the accuracy of the computed muscle forces. By using EMG activation patterns to constrain the muscle force estimations, we limited the error in predicting the timing of activations. Additionally, we used a generic model and not a subjectspecific modelling approach that incorporates each subject's lower limb anatomy.

\section{CONCLUSION}

Symmetry in performance of single leg hop distance masks important deficits in knee joint work in male athletes after ACLR. Specifically, they appear to underload their reconstructed knee during landing (while compensating at their hip) and overload their uninjured knee compared with healthy controls.

Correction notice This article has been corrected since it published Online First. Table 2 has been corrected.

Twitter Argyro Kotsifaki @RoulaKotsifaki, RodWhiteley @RodWhiteley, Sam Van Rossom @SamVanRossom, Vasileios Korakakis@KorakakisV, Roald Bahr @RoaldBahr, Vasileios Sideris @vasilisbme, Philip Graham-Smith @PhilipGrahamSm2 and Ilse Jonkers @IJonkers

Acknowledgements The authors thank the physiotherapists (ACL Group) of Aspetar Rehabilitation Department for assisting with subjects' recruitment.

Contributors AK, RW, SVR and IJ participated in the design and conception of the study. AK, VS and PG-S were responsible for data collection. AK, RW and VK performed the data analysis and table designs and all the authors contributed to the interpretation. AK drafted the manuscript and all the authors revised it critically and gave their approval of the final version.

Funding The authors have not declared a specific grant for this research from any funding agency in the public, commercial or not-for-profit sectors.

Competing interests None declared.

Patient consent for publication Not required.

Ethics approval Ethics approval was granted from the Anti-Doping Lab Qatar Institutional Review Board (F2017000227).

Provenance and peer review Not commissioned; externally peer reviewed.

Data availability statement Data are available on reasonable request. All publicly available data are included in the article or uploaded as online supplemental information.

Supplemental material This content has been supplied by the author(s). It has not been vetted by BMJ Publishing Group Limited (BMJ) and may not have been peer-reviewed. Any opinions or recommendations discussed are solely those of the author(s) and are not endorsed by BMJ. BMJ disclaims all liability and responsibility arising from any reliance placed on the content. Where the content includes any translated material, BMJ does not warrant the accuracy and reliability of the translations (including but not limited to local regulations, clinical guidelines, terminology, drug names and drug dosages), and is not responsible for any error and/or omissions arising from translation and adaptation or otherwise.

\section{ORCID iDs}

Argyro Kotsifaki http://orcid.org/0000-0002-7902-9206

Rod Whiteley http://orcid.org/0000-0002-1452-6228

Sam Van Rossom http://orcid.org/0000-0002-3206-9796

Vasileios Korakakis http://orcid.org/0000-0002-8033-3934

Roald Bahr http://orcid.org/0000-0001-5725-4237

Ilse Jonkers http://orcid.org/0000-0001-7611-3747

\section{REFERENCES}

1 Barber SD, Noyes FR, Mangine RE, et al. Quantitative assessment of functional limitations in normal and anterior cruciate ligament-deficient knees. Clin Orthop Relat Res 1990;255:204-14.

2 Barber-Westin SD, Noyes FR. Factors used to determine return to unrestricted sports activities after anterior cruciate ligament reconstruction. Arthroscopy 2011;27:1697-705.

3 Grindem H, Snyder-Mackler L, Moksnes H, et al. Simple decision rules can reduce reinjury risk by $84 \%$ after $A C L$ reconstruction: the Delaware-Oslo $A C L$ cohort study. $B r$ J Sports Med 2016;50:804-8.

4 Kyritsis $\mathrm{P}$, Bahr R, Landreau $\mathrm{P}$, et al. Likelihood of $\mathrm{ACL}$ graft rupture: not meeting six clinical discharge criteria before return to sport is associated with a four times greater risk of rupture. Br J Sports Med 2016;50:946-51.

5 Noyes FR, Barber SD, Mangine RE. Abnormal lower limb symmetry determined by function hop tests after anterior cruciate ligament rupture. Am J Sports Med 1991:19:513-8.

6 van Melick N, van Cingel REH, Brooijmans F, et al. Evidence-Based clinical practice update: practice guidelines for anterior cruciate ligament rehabilitation based on a systematic review and multidisciplinary consensus. Br J Sports Med 2016;50:1506-15.

7 Gokeler A, Welling W, Benjaminse A, et al. A critical analysis of limb symmetry indices of hop tests in athletes after anterior cruciate ligament reconstruction: a case control study. Orthop Traumatol Surg Res 2017;103:947-51.

8 Thomeé R, Neeter C, Gustavsson A, et al. Variability in leg muscle power and hop performance after anterior cruciate ligament reconstruction. Knee Surg Sports Traumatol Arthrosc 2012;20:1143-51.

9 Paterno MV, Rauh MJ, Schmitt LC, et al. Incidence of second ACL injuries 2 years after primary ACL reconstruction and return to sport. Am J Sports Med 2014;42:1567-73.

10 Webster KE, Feller JA. Exploring the high Reinjury rate in younger patients undergoing anterior cruciate ligament reconstruction. Am J Sports Med 2016;44:2827-32.

11 Wiggins AJ, Grandhi RK, Schneider DK, et al. Risk of secondary injury in younger athletes after anterior cruciate ligament reconstruction: a systematic review and meta-analysis. Am J Sports Med 2016;44:1861-76.

12 Ardern $\mathrm{CL}$, Webster $\mathrm{KE}$, Taylor NF, et al. Return to the preinjury level of competitive sport after anterior cruciate ligament reconstruction surgery: two-thirds of patients have not returned by 12 months after surgery. Am J Sports Med 2011;39:538-43.

13 Bahr R, Clarsen B, Ekstrand J. Why we should focus on the burden of injuries and illnesses, not just their incidence. Br J Sports Med 2018;52:1018-21.

14 Abrams GD, Harris JD, Gupta AK, et al. Functional performance testing after anterior cruciate ligament reconstruction. Orthop J Sports Med 2014;2:232596711351830.

15 Hegedus EJ, McDonough S, Bleakley C, et al. Clinician-friendly lower extremity physical performance measures in athletes: a systematic review of measurement properties and correlation with injury, part 1. The tests for knee function including the hop tests. Br J Sports Med 2015;49:642-8. 
16 Kotsifaki A, Korakakis V, Whiteley R, et al. Measuring only hop distance during single leg hop testing is insufficient to detect deficits in knee function after $\mathrm{ACL}$ reconstruction: a systematic review and meta-analysis. Br J Sports Med 2020:54:139-53.

17 Tanner R, Gore C. Physiological tests for elite athletes. Human kinetics, 2012

18 Dijkstra HP, Pollock N, Chakraverty R, et al. Return to play in elite sport: a shared decision-making process. Br J Sports Med 2017;51:419-20.

19 Davis RB, Õunpuu S, Tyburski D, et al. A gait analysis data collection and reduction technique. Hum Mov Sci 1991;10:575-87.

20 Duffell LD, Hope N, McGregor AH. Comparison of kinematic and kinetic parameters calculated using a cluster-based model and Vicon's plug-in gait. Proc Inst Mech Eng H 2014:228:206-10.

21 Hermens HJ, Freriks B, Disselhorst-Klug C, et al. Development of recommendations for SEMG sensors and sensor placement procedures. J Electromyogr Kinesiol 2000;10:361-74.

22 Bley AS, Correa JCF, Dos Reis AC, Reis ACD, et al. Propulsion phase of the single leg triple hop test in women with patellofemoral pain syndrome: a biomechanical study. PLoS One 2014;9:e97606.

23 Navacchia A, Ueno R, Ford KR, et al. EMG-Informed musculoskeletal modeling to estimate realistic knee anterior shear force during drop vertical jump in female athletes. Ann Biomed Eng 2019;47:2416-30.

24 van Melick N, Meddeler BM, Hoogeboom TJ, et al. How to determine leg dominance: the agreement between self-reported and observed performance in healthy adults. PLoS One 2017;12:e0189876.

25 Cole GK, Nigg BM, Ronsky JL, et al. Application of the joint coordinate system to three-dimensional joint attitude and movement representation: a standardization proposal. J Biomech Eng 1993;115:344-9.

26 Bell AL, Brand RA, Pedersen DR. Prediction of hip joint centre location from external landmarks. Hum Mov Sci 1989;8:3-16.

27 Catelli DS, Wesseling $\mathrm{M}$, Jonkers I, et al. A musculoskeletal model customized for squatting task. Comput Methods Biomech Biomed Engin 2019;22:21-4.

28 Delp SL, Anderson FC, Arnold AS, et al. OpenSim: open-source software to create and analyze dynamic simulations of movement. IEEE Trans Biomed Eng 2007; $54: 1940-50$

$29 \mathrm{Xu} \mathrm{H}$, Bloswick D, Merryweather A. An improved OpenSim gait model with multiple degrees of freedom knee joint and knee ligaments. Comput Methods Biomech Biomed Engin 2015;18:1217-24.

30 Konrath JM, Saxby DJ, Killen BA, et al. Muscle contributions to medial tibiofemoral compartment contact loading following $\mathrm{ACL}$ reconstruction using semitendinosus and gracilis tendon grafts. PLoS One 2017;12:e0176016.

31 Mokhtarzadeh H, Perraton L, Fok L, et al. A comparison of optimisation methods and knee joint degrees of freedom on muscle force predictions during single-leg hop landings. J Biomech 2014;47:2863-8.

32 Wesseling $M$, Kainz $H$, Hoekstra $T$, et al. Botulinum toxin injections minimally affect modelled muscle forces during gait in children with cerebral palsy. Gait Posture 2020;82:54-60

33 Suydam SM, Manal K, Buchanan TS. The Advantages of Normalizing Electromyography to Ballistic Rather than Isometric or Isokinetic Tasks. J Appl Biomech 2017;33:189-96.

34 Orishimo KF, Kremenic IJ, Mullaney MJ, et al. Adaptations in single-leg hop biomechanics following anterior cruciate ligament reconstruction. Knee Surg Sports Traumatol Arthrosc 2010;18:1587-93.

35 Wren TAL, Mueske NM, Brophy $\mathrm{CH}$, et al. Hop distance symmetry does not indicate normal landing biomechanics in adolescent athletes with recent anterior cruciate ligament reconstruction. J Orthop Sports Phys Ther 2018;48:622-9.

36 Shapiro SS, Wilk MB. An analysis of variance test for normality (complete samples). Biometrika 1965;52:591-611.

37 Brown MB, Forsythe AB. Robust tests for the equality of variances. J Am Stat Assoc 1974:69:364-7.

38 Wilcoxon F. Individual comparisons by ranking methods. breakthroughs in statistics. Springer, 1992: 196-202.

39 Mann HB, Whitney DR. On a test of whether one of two random variables is stochastically larger than the other. Ann. Math. Statist. 1947;18:50-60.

40 Cohen J. Statistical power analysis for the behavioral sciences. Academic press, 2013

41 Hedges LV, Olkin I. Statistical methods for meta-analysis. Academic press, 2014.

42 Logerstedt D, Grindem H, Lynch A, et al. Single-legged hop tests as predictors of selfreported knee function after anterior cruciate ligament reconstruction: the DelawareOslo ACL cohort study. Am J Sports Med 2012;40:2348-56.
43 Ross MD. The relationship between functional levels and fear-avoidance beliefs following anterior cruciate ligament reconstruction. J Orthop Traumatol 2010;11:237-43.

44 Irrgang JJ, Anderson AF, Boland AL, et al. Development and validation of the International knee documentation Committee subjective knee form. Am J Sports Med 2001;29:600-13.

45 Webster KE, Feller JA, Lambros C. Development and preliminary validation of a scale to measure the psychological impact of returning to sport following anterior cruciate ligament reconstruction surgery. Phys Ther Sport 2008;9:9-15.

46 Ebert JR, Webster KE, Edwards PK, et al. Current perspectives of Australian therapists on rehabilitation and return to sport after anterior cruciate ligament reconstruction: $A$ survey. Phys Ther Sport 2019;35:139-45.

47 Nagai T, Schilaty ND, Laskowski ER, et al. Hop tests can result in higher limb symmetry index values than isokinetic strength and leg press tests in patients following $\mathrm{ACL}$ reconstruction. Knee Surg Sports Traumatol Arthrosc 2020;28:816-22.

48 Palmieri-Smith RM, Lepley LK. Quadriceps strength asymmetry after anterior cruciate ligament reconstruction alters knee joint biomechanics and functional performance at time of return to activity. Am J Sports Med 2015;43:1662-9.

49 Schmitt LC, Paterno MV, Hewett TE. The impact of quadriceps femoris strength asymmetry on functional performance at return to sport following anterior cruciate ligament reconstruction. J Orthop Sports Phys Ther 2012;42:750-9.

50 King E, Richter C, Franklyn-Miller A, et al. Whole-body biomechanical differences between limbs exist 9 months after ACL reconstruction across jump/landing tasks. Scand J Med Sci Sports 2018:28:2567-78.

51 Losciale JM, Bullock G, Cromwell C, et al. Hop testing lacks strong association with key outcome variables after primary anterior cruciate ligament reconstruction: a systematic review. Am J Sports Med 2020;48:511-22.

52 Mattacola CG, Perrin DH, Gansneder BM, et al. Strength, functional outcome, and postural stability after anterior cruciate ligament reconstruction. J Athl Train 2002;37:262-8

53 Smeets A, Verheul J, Vanrenterghem J, et al. Single-Joint and whole-body movement changes in anterior cruciate ligament athletes returning to sport. Med Sci Sports Exerc 2020:52:1658-67.

54 Paterno MV, Ford KR, Myer GD, et al. Limb asymmetries in landing and jumping 2 years following anterior cruciate ligament reconstruction. Clin J Sport Med 2007; 17:258-62

55 Zabala ME, Favre J, Scanlan SF, et al. Three-Dimensional knee moments of ACL reconstructed and control subjects during gait, stair ascent, and stair descent. $J$ Biomech 2013:46:515-20.

56 Wellsandt E, Failla MJ, Snyder-Mackler L. Limb symmetry indexes can overestimate knee function after anterior cruciate ligament injury. J Orthop Sports Phys Ther 2017:47:334-8.

57 Sigward SM, Chan M-SM, Lin PE, et al. Compensatory strategies that reduce knee extensor demand during a bilateral squat change from 3 to 5 months following anterior cruciate ligament reconstruction. J Orthop Sports Phys Ther 2018;48:713-8.

58 Patras K, Zampeli F, Ristanis S, et al. Hamstring-dominant strategy of the bone-patellar tendon-bone graft anterior cruciate ligament-reconstructed leg versus quadricepsdominant strategy of the contralateral intact leg during high-intensity exercise in male athletes. Arthroscopy 2012;28:1262-70.

59 Lie MM, Risberg MA, Storheim K, et al. What's the rate of knee osteoarthritis 10 years after anterior cruciate ligament injury? an updated systematic review. $\mathrm{Br}$ J Sports $\mathrm{Med}$ 2019;53:1162-7

60 Donnelly CJ, Chinnasee C, Weir G, et al. Joint dynamics of rear- and fore-foot unplanned sidestepping. J Sci Med Sport 2017;20:32-7

61 Stearne SM, Alderson JA, Green BA, et al. Joint kinetics in rearfoot versus forefoot running: implications of switching technique. Med Sci Sports Exerc 2014;46:1578-87.

62 Azmi NL, Ding Z, Xu R, et al. Activation of biceps femoris long head reduces tibiofemoral anterior shear force and tibial internal rotation torque in healthy subjects. PLoS One 2018:13:e0190672.

63 Maniar N, Schache AG, Sritharan P, et al. Non-knee-spanning muscles contribute to tibiofemoral shear as well as valgus and rotational joint reaction moments during unanticipated sidestep cutting. Sci Rep 2018;8:2501.

64 Paterno MV, Huang B, Thomas S, et al. Clinical factors that predict a second ACL injury after $A C L$ reconstruction and return to sport: preliminary development of a clinical decision algorithm. Orthop J Sports Med 2017;5:232596711774527.

65 DeLang MD, Rouissi M, Bragazzi NL, et al. Soccer Footedness and Between-Limbs muscle strength: systematic review and meta-analysis. Int I Sports Physiol Perform 2019:14:551-62. 